\title{
Cloud Resource Optimization System Based on Time and Cost
}

\author{
Bhupesh Kumar Dewangan \\ School of Computer Science and Engineering, \\ University of Petroleum and Energy Studies, Dehradun, India. \\ Corresponding author: bhupesh.dewangan@gmail.com \\ Amit Agarwal \\ School of Computer Science and Engineering, \\ University of Petroleum and Energy Studies, Dehradun, India. \\ E-mail: aagarwal@ddn.upes.ac.in \\ Tanupriya Choudhury \\ School of Computer Science and Engineering, \\ University of Petroleum and Energy Studies, Dehradun, India. \\ E-mail: tanupriya1986@gmail.com \\ Ashutosh Pasricha \\ Schlumberger Pvt. Ltd, New Delhi, India. \\ E-mail: apasricha@slb.com
}

(Received August 2, 2019; Accepted March 31, 2020)

\begin{abstract}
Resource management in cloud could be a time and cost-effective activity if it is managed property. These resources are accessible and computable which is totally dependent upon the management techniques applied in cloud. In a cloud setting, heterogeneous, vulnerability, and scattering of resources creates many issues of distribution among the workloads which need to be compute. Specialists still face inconveniences to pick the prudent, material and expend less time to execution of resource portion to the cloud. This investigation delineates an expansive composed writing examination of asset administration inside the space of cloud typically and cloud asset administration based on SLA with multi-objective functions like cost and time. In this paper, an autonomic cloud resource-management technique is proposed to resolve identified issues by adopting the self-characteristics mechanism and improved Antlion optimization algorithm and tested in cloudsim toolkit and Aws Ec2 environment. The implementation results of proposed work are the evidence that it is better performing as compared with the existing frameworks, however, the performance evaluation method depends upon the different cloud environment and it may vary.
\end{abstract}

Keywords- Cloud computing, Autonomic computing, Self-optimization, Fuzzy, Resource scheduling.

\section{Introduction}

Cloud server and virtual machine perform significant jobs to manage resources in cloud. The utilization of resources (Sutar et al., 2020) is additionally high gratitude to a great deal of execution time and resource failure. To deal with the resource in cloud quickly, the energy consumption use should be minimize and avoid the resource failure. The resource computer equipment plans the approaching cloud remaining tasks upheld the outstanding burdens subtle elements. To begin with, get cloud remaining tasks to plan, so acknowledge material, and available assets and cloud outstanding burdens mapped quickly upheld the arranging approaches. Dispatcher is utilize to dispatch the outstanding tasks at hand for execution (Levitin et al., 2020). The work is send exclusively, if the remaining tasks will be dead about the quality of service QoS parameters said in 
International Journal of Mathematical, Engineering and Management Sciences

Vol. 5, No. 4, 758-768, 2020

https://doi.org/10.33889/IJMEMS.2020.5.4.060

service level agreement SLA. Asset screen is utilize to discover the remaining of arranging of resource like regardless of whether the coveted scope of resource is given or not. QoS screen contains the learning with respect to QoS parameters to discover, regardless of whether every one of the outstanding tasks at hand square measure execution among their particular fluctuate or not. Assume point in time could be a QoS parameter, in this manner duty of QoS screen is to learn regardless of whether outstanding tasks at hand square measure dead before wanted point in time or not. There is infringement of SLA (Dewangan et al., 2018) if work executes once wanted point in time. Streamlining of vitality in cloud asset arranging is about of practicable strategy that limit the vitality utilization and expand the virtual machine (VM) use. On the whole, the strength of asset designation is improve through vitality enhancement. This technique limits the execution time, SLA infringement rate, and worth that expansion the framework execution. The present streamlining ways square measure action static conduct like Genetic-Algorithm, Particle-swarmadvancement rule, Ant-settlement principle and microorganism chase improvement. The static conduct of the framework could wind up in moderate in execution and high in worth. The framework needs the keen framework to advance the vitality and diverse target capacities. This clever method will manufacture the dynamic advancement structures, which improve vitality also as worth and time. The dynamic advancement while not human intercession is execute without anyone else's input qualities. In this manner, vitality is self-streamlined (Chen et al., 2020) and piece. It will precisely understand the best goals for asset provisioning. Adaptation to non-critical failure advancement is an ability of a PC structure, electronic structure or framework to pass on ceaseless organization, regardless of at least one in the entirety of its parts growing short. Adjustment to non-basic disappointment in like manner settle potential organization obstruction known with programming or guideline botches. The reason for existing is to balance dark disillusionment that may happen because of a singular motivation behind disappointment. Faulttolerant (Dewangan et al., 2016) frameworks shall compose for numerous disappointments. Such structures usually distinguish a disappointment of the computer processor unit, I/O system, memory cards, motherboard, and management provide or framework components. The frustration purpose is recognized, and a support fragment or technique during a flash has its spot with no loss of organization. To make sure adjustment to non-basic disappointment, tries desirous to purchase a load of masterminded computer instrumentality and a helper uninterruptible power provide device. The target is to stay the crash of key frameworks and systems, concentrating on problems known with up time and period (Dewangan et al., 2019). Adaptation to internal failure is offerprogramming put in instrumentality, or by some mixture of the two. In this research work, the cloud resources are allocating to the workloads submitted by cloud user based on service level agreement (SLA). The multi-objective function like cost and time are main concern of this research. The proposed system is simulating and evaluating based on above multi-objective parameters and the results are obtain in terms of cost and time, which compared with other existing frameworks, observed the utmost performance.

\section{Related Work}

A state of art survey has been conduct on SLA, cost and based resource management and illustrating as follows:

\subsection{SLA-Based Resource Management}

Resource planning supported SLA has been conduct by following papers, automatic SLA-aware asset arranging algorithmic program to decrease SLA encroachment and violation rate that works in cloud virtual condition to execute benefit while not infringement of SLA. This outline joins three parts: benefit agent, understanding arrange and request preparing to execute benefit while not SLA 
International Journal of Mathematical, Engineering and Management Sciences

Vol. 5, No. 4, 758-768, 2020

https://doi.org/10.33889/IJMEMS.2020.5.4.060

infringement (Kertesz et al., 2011). QoS based resource-arranging system to estimate the execution underneath disparate asset assignment by resize of VM. Multi-Workflows essentially based arranging strategy to improve the arranging achievement rate together with elective QoS parameters. It settles on cloud providers and learning focuses in an exceedingly multi-cloud climate as an administration supervisor upheld QoS parameters (Xu et al., 2009). Execution of the hatchling is screen by Spe-QuloS in discontinuous way, resources are gave only if there ought to emerge an event of benefit necessities capably, and SpeQuloS upgrades quality of execution and envision the execution time of occupation (Delamare et al., 2012). Random-Key Genetic algorithmic program fundamentally based orchestrating game plan to diminish execution time and running expenses and enhance quantifiability. This algorithmic program settled a substitution numerous composite net administration asset designation and arranging disadvantage in an exceedingly half breed cloud situation wherever there are additionally limited local assets from individual mists and various out their re-sources from open mists (Ai et al., 2011).

\subsection{Cost-Based Resource Management}

Resource arranging bolstered cost led by following papers. Imperative asset arranging algorithmic run for pack of assignments amid which undertaking is picked bolstered FCFS philosophy. This system limits value, fulfillment time and enhances C.P.U. execution nevertheless, because of drawback of starvation, this instrument is not successful (Oprescu and Kielmann, 2010). Contemplated the enhancement drawback forcing condition like execution of occupation isn't preemptible and point constrained amid a multi-supplier crossover cloud surroundings bolstered the needs of data transmission, C.P.U. furthermore, memory, the order of non-supplier relocate capable outstanding burdens is finished (Van et al., 2010). DAG (Directed Acyclic Graph) based for the most part assignment arranging instrument to downsize cost and Makespan exploitation two heuristic ways. First system maps errands to the preeminent effective virtual assets exploitation humanist predominance and second procedure is utilize to diminish the money related costs of nonbasic assignment in true applications (Su et al., 2013). Bestowed VM based for the most part asset arranging procedure to measure the general estimation of Gang arranging with starvation dealing with, movements, and execution of superior venture applications. To contact upon starvation in arranging procedure, organized line is utilized to search out the need of every application bolstered their coveted point and so forth (Moschakis and Karatza, 2011).

\subsection{Time-Based Resource Management}

Resource programming supported time has been conduct by following papers. Asset programming strategy to reduce data exchange and process costs, arrange data measure and vitality utilization. Execution of this algorithmic control is assessed exploitation unique execution parameters like scope of lost due dates, esteem sparing and process strength and break down the effect of estimation mistakes on execution (Van et al., 2013). Programming algorithmic lead to anticipate asset needs of occupation exploitation adjusted machine learning based for the most part indicator and fast explanatory indicator and show that anyway this programming algorithmic run is utilize to foresee asset needs to reduce SLA infringement (Reig et al., 2010). Bestowed Partial vital ways based for the most part ICPCP (Abrishami and Naghibzadeh, 2012) IaaS Cloud Partial essential Paths and IC-PCP with point Distribution (IC-PCPD2) to arrangement and timetable enormous work processes. The calculation time is lesser amid this methodology in any case; this is regularly frail to live measurable execution and UTC precisely. Vitality is amount of vitality devoured by an asset to end the execution of work. Esteem is an amount of significant worth will pay in one hour for the execution of work. In time based for the most part asset programming, client and provider talk over 
International Journal of Mathematical, Engineering and Management Sciences

Vol. 5, No. 4, 758-768, 2020

https://doi.org/10.33889/IJMEMS.2020.5.4.060

QoS parameters inside the style of composed material alluded to as SLA. The comparative analysis is presented in Table 1.

Table 1. Comparative analysis of SLA, Cost and Time-based resource management techniques

\begin{tabular}{|c|c|c|c|c|c|c|c|c|}
\hline System & ET & Cost & $\overline{\mathrm{EE}}$ & $\overline{\mathrm{RU}}$ & $\overline{\mathrm{OC}}$ & SLA & QoS & $\overline{\mathrm{CE}}$ \\
\hline (Sutar et al., 2020) & & & & $\sqrt{ }$ & & $\sqrt{1}$ & & \\
\hline (Levitin et al., 2020) & $\sqrt{ }$ & & & & $\sqrt{ }$ & & & \\
\hline (Chen et al., 2020) & & & $\sqrt{ }$ & & & & & \\
\hline (Delamare et al., 2012) & & $\sqrt{ }$ & $\sqrt{ }$ & & & & $\sqrt{ }$ & \\
\hline (Ai et al., 2011) & & $\sqrt{ }$ & & $\sqrt{ }$ & & & & \\
\hline (Oprescu and Kielmann, 2010) & & $\sqrt{ }$ & & & & & & \\
\hline (Van et al., 2010) & & $\sqrt{ }$ & & & $\sqrt{ }$ & & & $\sqrt{ }$ \\
\hline (Su et al., 2013) & $\sqrt{ }$ & $\sqrt{ }$ & $\sqrt{ }$ & & & $\sqrt{ }$ & $\sqrt{ }$ & \\
\hline (Moschakis and Karatza, 2011) & $\sqrt{ }$ & & $\sqrt{ }$ & & & & $\sqrt{ }$ & \\
\hline (Van et al., 2013) & $\sqrt{ }$ & $\sqrt{ }$ & & & & $\sqrt{ }$ & $\sqrt{ }$ & \\
\hline (Reig et al., 2010) & $\sqrt{ }$ & $\sqrt{ }$ & & & & & & \\
\hline
\end{tabular}

\section{Optimization Method}

In this research, antlion optimizer (ALO) (Mirjalili, 2015) is used for the fittest VM selection. This algorithm is also used in healthcare, construction and civil engineering to find the optimal solutions. According to this, The ALO imitates collaboration among virtual machines VM's and tasks D in the system. To demonstrate such collaborations, task D are required to submit over the pursuit system, and VM's are permitted to chase them and allot to compute the task D.

\subsection{Parameters}

$z(t)=$ Random walk function,

$n=$ maximum number of iteration,

cumsum = calculate the cumulative sum

$d(t)=$ stochasticfunction,

$t=$ step of random walk,

$A_{D}=$ position of each ant,

$A_{o D}=$ Stores fitness value of each ant,

$A_{V M}=$ position of each antlon,

$A_{\text {oVM }}=$ Stores fitness value od each antlion

\subsection{Mathematical Model}

Since ants move stochastically in nature when searching for food, a random walk is chosen for modelling ants' movement as follows:

Since task D submitted stochastically in nature while looking for any computing resources like $\mathrm{VM}$, an irregular walk is picked for displaying task D development as pursues and presented in eq. (1):

$z(t)=\left[0\right.$, cumsum $\left(2 d\left(t_{1}-1\right)\right.$, cumsum $\left(2 d\left(t_{2}-1\right)\right.$, cumsum $\left(2 d\left(t_{3}-1\right) \ldots \ldots\right.$ cumsum $\left(2 d\left(t_{n}-1\right)\right.$,

Computing time span can be presented in eq. (2): 
International Journal of Mathematical, Engineering and Management Sciences

Vol. 5, No. 4, 758-768, 2020

https://doi.org/10.33889/IJMEMS.2020.5.4.060

$d(t)=\left\{\begin{array}{l}1 \text { if random }>0.5 \\ 0 \text { if random }<0.5\end{array}\right.$

The random task D is allocated to virtual machine VM and fitness of each VM is obtained through CPU, RAM, and Bandwidth Utilization for each VM's. The following optimization equation is used to store the position of task $\mathrm{D}$ and presented in eq. (3):

$$
A_{D}=\begin{array}{lll}
A_{11} & A_{12} & \ldots A_{1 n} \\
A_{21} & A_{22} & \ldots A_{2 n} \\
A_{n 1} & A_{n 2} & . . A_{n m}
\end{array}
$$

The following fitness function is used to store the fitness value of task D presented in eq. (4):

$$
A_{o D}=\begin{array}{lll}
f A_{11} & f A_{12} & . . f A_{1 n} \\
f A_{21} & f A_{22} & . . f A_{2 n} \\
f A_{n 1} & f A_{n 2} & \ldots f A_{n m}
\end{array}
$$

The following metric is used to restore the VM fitness values presented in eq. (5):

$$
A_{V M}=\begin{array}{lll}
A L_{11} & A L_{12} & \ldots A L_{1 n} \\
A L_{21} & A L_{22} & \ldots A L_{2 n} \\
A L_{n 1} & A L_{n 2} & \ldots A L_{n m}
\end{array}
$$

The following fitness function is used to store the fitness value of VM's and presented in eq. (6):

$$
A_{o V M}=\begin{array}{lll}
f A_{11} & f A_{12} & \ldots f A_{1 n} \\
f A_{21} & f A_{22} & . . f A_{2 n} \\
f A_{n 1} & f A_{n 2} & \ldots f A_{n m}
\end{array}
$$

The roulette wheel is used to select a best VM from its population based on multi objective fitness calculation (CPU, RAM, Bandwidth, and Resource Utilization). This highly fit VM to catch the task D with high probability.

The pseudocode of above method is as follows:

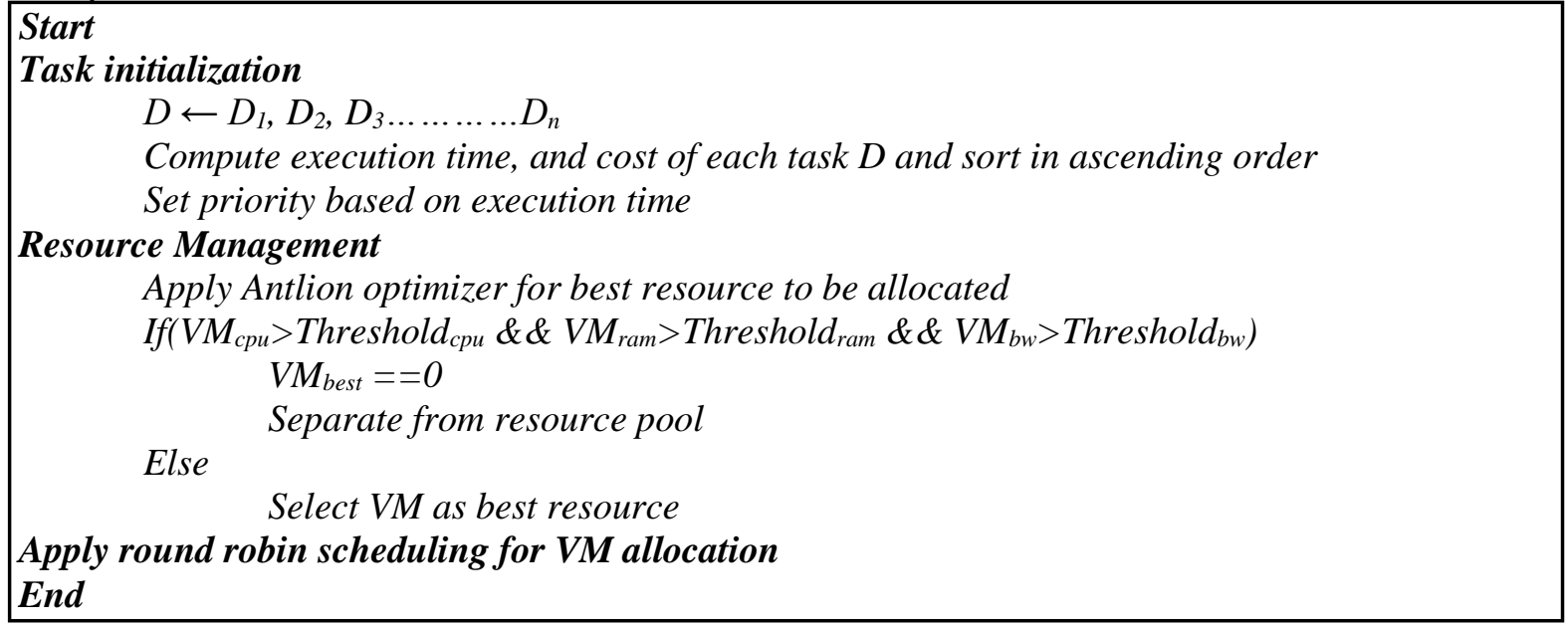




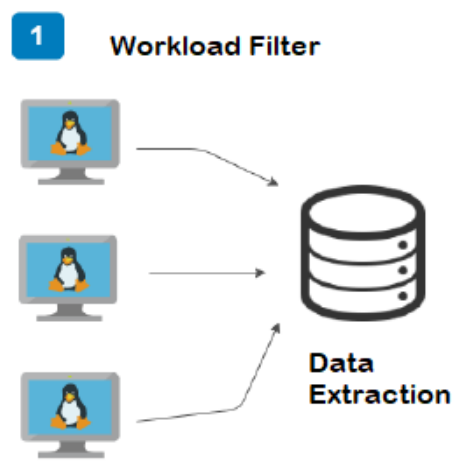

2 Fault-tolerant functions

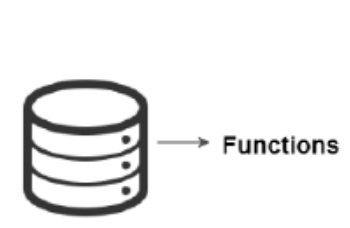

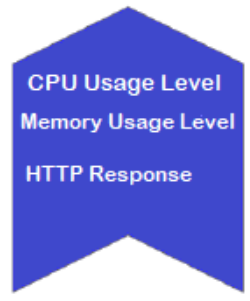

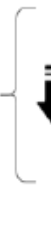

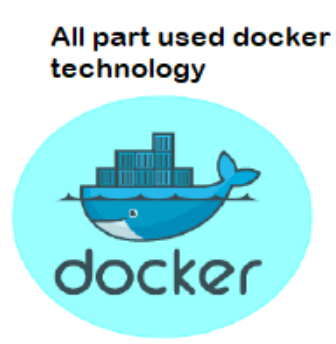

3

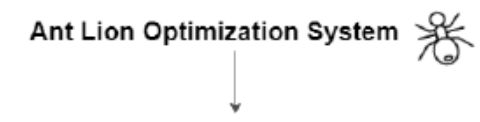

The algorithm can control the number of machines and make the decision

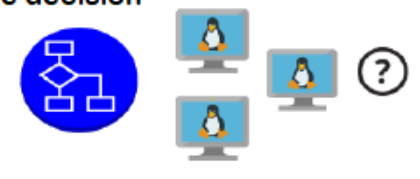

Storage in Amazon

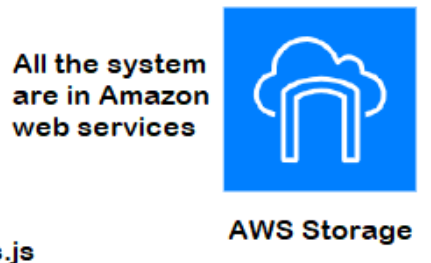

Figure 1. Architecture of Proposes ALO based solution

\section{Architecture}

The architecture of proposed system shown in Figure 1, is implemented and tested in AWS EC2 (Amazon Web Service Elastic Cloud Computing) dynamic environment. The submitted workloads are extracted and filtered through, then functions (CPU, Memory, and Bandwidth) applied to the EC2 instances which is now optimized through ALO and provide the optimal EC2 instance for scheduling the workloads to optimal EC2 instances. The novel approach is the consideration of Bandwidth function with CPU and Memory function to get the optimal EC2 instances.

\section{Results and Analysis}

The proposed system is simulated and tested in cloudsim and AWS EC2 environments with 20 VM's and 50-1500 task (population). The comparative analysis is done with SpeQuloS (Delamare et al., 2012) and Oces (Xu et al., 2009). The observations of the test results are as discussed below:

\subsection{Execution Time Analysis}

The execution time for proposed system is recorded on the basis of successfully scheduling of VM to workloads submitted by cloud user, in this, the systems tested with eight sample data. First the systems generate synthesis test data which is set of 50 to 1500 set of 30 task D. It is defined as the product of total execution time to schedule of tasks to optimal resources (VM). The proposed system scheduled the 50 to 1500 task D successfully and the average time is observed as 960 milliseconds. Whereas the other existing works also be tested in same environment $t$ with same number of tasks and the average time for scheduling of given task is observed as 1061 and 1140 milliseconds, which shows that the proposed system take $9 \%$ less time for scheduling with best VM's. The comparative analysis is presented in Figure 2. The overall analysis of proposed work 
International Journal of Mathematical, Engineering and Management Sciences

Vol. 5, No. 4, 758-768, 2020

https://doi.org/10.33889/IJMEMS.2020.5.4.060

based on execution time is evidence of better performance as compared with SpeQulos and Oces. However, the claim may differ the platform other than Asw Ec2 and Cloudsim toolkit.

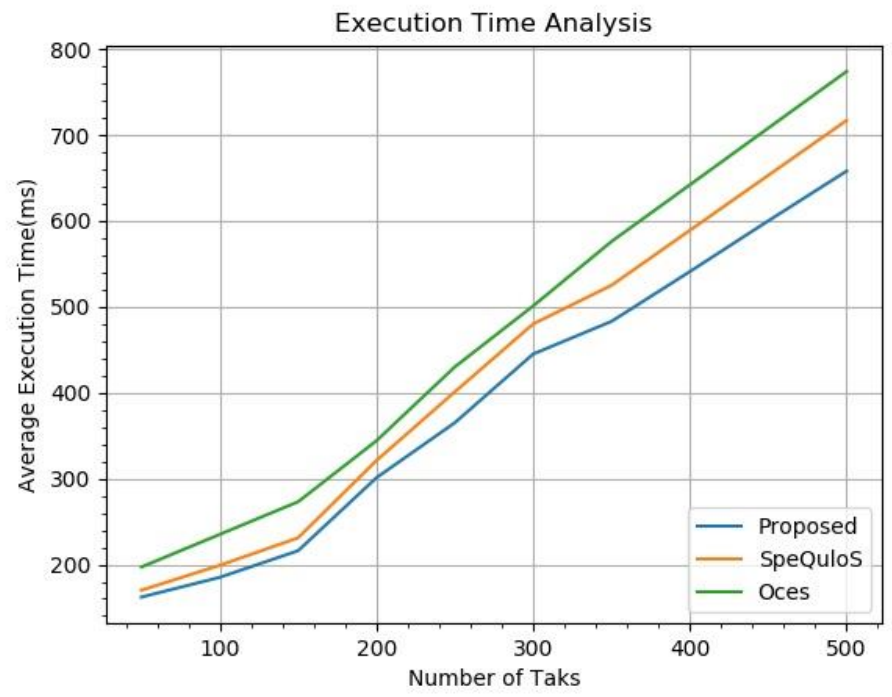

Figure 2. Execution Time Analysis of proposed work (ms)

\subsection{Cost Analysis}

The cost for proposed system is recorded on the basis of successfully scheduling of VM to workloads submitted by cloud user. First the systems generate synthesis test data which is set of 50 to 1500 set of 30 task D. It is defined as the product of total execution time to schedule of tasks to optimal resources (VM). The proposed system scheduled the 50 to 1500 task D successfully and the average cost is observed as $\$ 2.8 \mathrm{~K}$. Whereas the other existing works also be tested in same environment $\mathrm{t}$ with same number of tasks and the average cost for scheduling of given task is observed as $\$ 3.1 \mathrm{~K}$ and $\$ 3.4 \mathrm{~K}$, which shows that the proposed system take 5\% less cost for scheduling with best VM's. The comparative analysis is presented in Figure 3. The overall analysis of proposed work based on cost is evidence of better performance as compared with SpeQulos and Oces. However, the claim may differ the platform other than Asw Ec2 and Cloudsim toolkit. 
International Journal of Mathematical, Engineering and Management Sciences

Vol. 5, No. 4, 758-768, 2020

https://doi.org/10.33889/IJMEMS.2020.5.4.060

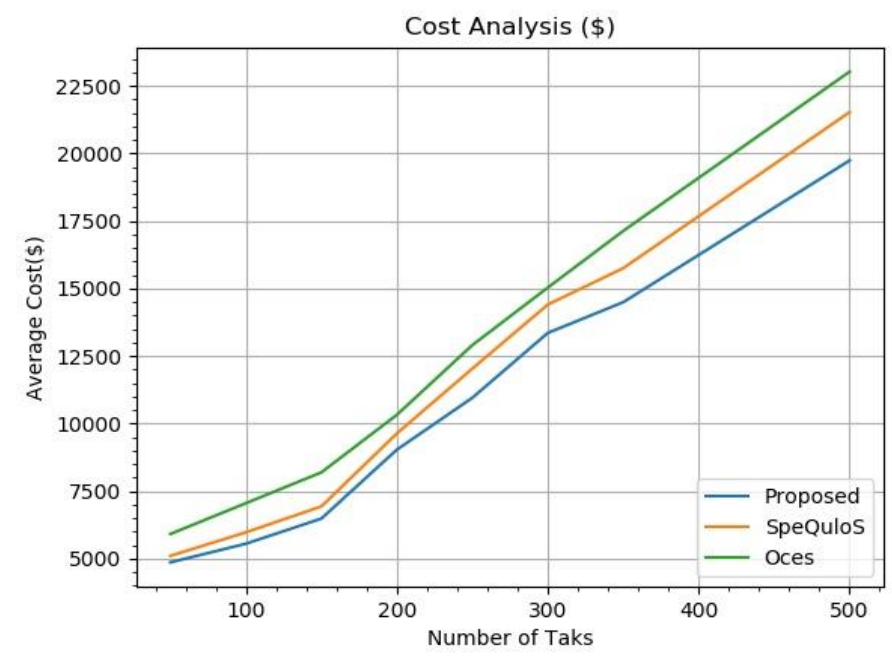

Figure 3. Cost Analysis of proposed work (ms)

\subsection{SLA Violation Rate Analysis}

The proposed system scheduled the 50 to 1500 task D successfully and the average SLA violation rate is observed as 0.42 milliseconds. Whereas the other existing works also be tested in same environment with same number of tasks and the average SLA violation rate for scheduling of given task is observed as 0.82 and 0.85 milliseconds, which shows that the proposed system take $48 \%$ less for scheduling with best VM's. The comparative analysis is presented in Figure 4. The overall analysis of proposed work based on SLA is evidence of better performance as compared with SpeQulos and Oces. However, the claim may differ the platform other than Asw Ec2 and Cloudsim toolkit.

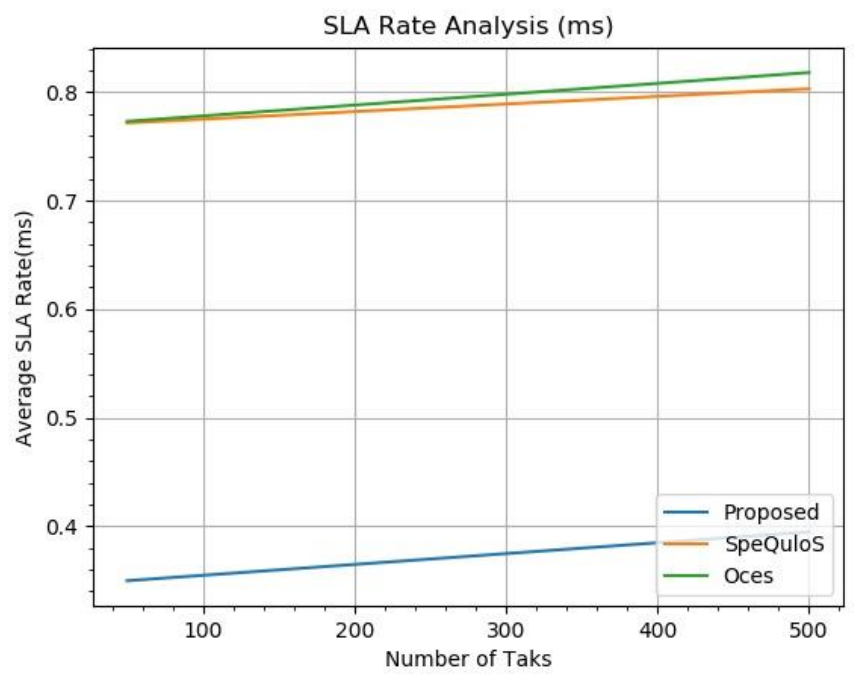

Figure 4. SLA Violation rate Analysis of proposed work (ms) 
International Journal of Mathematical, Engineering and Management Sciences

Vol. 5, No. 4, 758-768, 2020

https://doi.org/10.33889/IJMEMS.2020.5.4.060

\subsection{Observations}

Real-time cloud platform generally schedules the task based on their arrival time, moreover in this research, the fundamental approach is overruled and optimization-based policy has been introduced to select better and available resource to execute the desired task. The proposed system is tested in simulation as well as real-time environment and the data obtained after execution of proposed framework is presented in Table 2 .

Table 2. SLA, Cost and Execution time analysis of proposed system

\begin{tabular}{|c|c|c|c|c|c|c|c|c|c|}
\hline \multirow{2}{*}{ Workloads } & \multicolumn{3}{|c|}{ Execution Time Analysis (ms) } & \multicolumn{2}{|c|}{ Cost Analysis (\$) } & \multicolumn{4}{|c|}{ SLA Rate Analysis (ms) } \\
\hline & Proposed & SpeQulos & Oces & Proposed & SpeQulos & Oces & Proposed & SpeQulos & Oces \\
\hline 50 & 162 & 170 & 197 & 4860 & 5100 & 5910 & 0.3497 & 0.7715 & 0.773 \\
\hline 100 & 185 & 199 & 235 & 5550 & 5970 & 7050 & 0.3547 & 0.7750 & 0.778 \\
\hline 150 & 216 & 231 & 273 & 6480 & 6930 & 8190 & 0.3597 & 0.7785 & 0.783 \\
\hline 200 & 301 & 321 & 344 & 9030 & 9630 & 10320 & 0.3647 & 0.7820 & 0.788 \\
\hline 250 & 365 & 401 & 430 & 10950 & 12030 & 12900 & 0.3697 & 0.7855 & 0.793 \\
\hline 300 & 445 & 480 & 501 & 13350 & 14400 & 15030 & 0.3747 & 0.7890 & 0.798 \\
\hline 350 & 483 & 525 & 576 & 14490 & 15748 & 17127 & 0.3797 & 0.7925 & 0.803 \\
\hline 400 & 541 & 589 & 642 & 16238 & 17673 & 19092 & 0.3847 & 0.7960 & 0.808 \\
\hline 450 & 600 & 653 & 708 & 17987 & 19598 & 21057 & 0.3897 & 0.7995 & 0.813 \\
\hline 500 & 658 & 717 & 774 & 19736 & 21524 & 23023 & 0.3947 & 0.8030 & 0.818 \\
\hline 550 & 716 & 782 & 840 & 21484 & 23449 & 24988 & 0.3997 & 0.8065 & 0.823 \\
\hline 600 & 774 & 846 & 906 & 23233 & 25374 & 26953 & 0.4047 & 0.8100 & 0.828 \\
\hline 650 & 833 & 910 & 973 & 24981 & 27299 & 28918 & 0.4097 & 0.8135 & 0.833 \\
\hline 700 & 891 & 974 & 1039 & 26730 & 29224 & 30883 & 0.4147 & 0.8170 & 0.838 \\
\hline 750 & 949 & 1038 & 1105 & 28478 & 31149 & 32848 & 0.4197 & 0.8205 & 0.843 \\
\hline 800 & 1008 & 1102 & 1171 & 30227 & 33074 & 34813 & 0.4247 & 0.8240 & 0.848 \\
\hline 850 & 1066 & 1167 & 1237 & 31975 & 35000 & 36779 & 0.4297 & 0.8275 & 0.853 \\
\hline 900 & 1124 & 1231 & 1303 & 33724 & 36925 & 38744 & 0.4347 & 0.8310 & 0.858 \\
\hline 950 & 1182 & 1295 & 1370 & 35472 & 38850 & 40709 & 0.4397 & 0.8345 & 0.863 \\
\hline 1000 & 1241 & 1359 & 1436 & 37221 & 40775 & 42674 & 0.4447 & 0.8380 & 0.868 \\
\hline 1050 & 1299 & 1423 & 1502 & 38970 & 42700 & 44639 & 0.4497 & 0.8415 & 0.873 \\
\hline 1100 & 1357 & 1487 & 1568 & 40718 & 44625 & 46604 & 0.4547 & 0.8450 & 0.878 \\
\hline 1150 & 1416 & 1552 & 1634 & 42467 & 46550 & 48569 & 0.4597 & 0.8485 & 0.883 \\
\hline 1200 & 1474 & 1616 & 1700 & 44215 & 48476 & 50535 & 0.4647 & 0.8520 & 0.888 \\
\hline 1250 & 1532 & 1680 & 1767 & 45964 & 50401 & 52500 & 0.4697 & 0.8555 & 0.893 \\
\hline 1300 & 1590 & 1744 & 1833 & 47712 & 52326 & 54465 & 0.4747 & 0.8590 & 0.898 \\
\hline 1350 & 1649 & 1808 & 1899 & 49461 & 54251 & 56430 & 0.4797 & 0.8625 & 0.903 \\
\hline 1400 & 1707 & 1872 & 1965 & 51209 & 56176 & 58395 & 0.4847 & 0.8660 & 0.908 \\
\hline 1450 & 1765 & 1937 & 2031 & 52958 & 58101 & 60360 & 0.4897 & 0.8695 & 0.913 \\
\hline 1500 & 1824 & 2001 & 2098 & 54707 & 60027 & 62326 & 0.4947 & 0.8730 & 0.918 \\
\hline
\end{tabular}


International Journal of Mathematical, Engineering and Management Sciences

Vol. 5, No. 4, 758-768, 2020

https://doi.org/10.33889/IJMEMS.2020.5.4.060

\section{Conclusion}

Resource management makes the cloud cost effective, where optimizing the resource is minimized the cost and time to be taken for scheduling and task to the workload. This research is focused on optimizing time, cost, and SLA rate so that the efficiency of the system increases. With this objective, the system is proposed, which is simulated and implemented in cloudsim as well as tested in Amazon web services AWS. The proposed work is managed the scheduling activity with low cost and time. The experimental results show that the proposed model is more efficient as compared with the existing systems. The less SLA rate is evidence of the proposed work that is more trustable on account of cloud end user. The analysis proofs that proposed system is more efficient based on cost, time and SLA rate.

\section{Conflict of Interest}

The authors confirm that there is no conflict of interest to declare for this publication.

\section{Acknowledgments}

This research did not receive any specific grant from funding agencies in the public, commercial, or not-for-profit sectors. The authors sincerely appreciate the editor and reviewers for their time and valuable comments.

\section{References}

Abrishami, S., \& Naghibzadeh, M. (2012). Deadline-constrained workflow scheduling in software as a service cloud. Scientia Iranica, 19(3), 680-689.

Ai, L., Tang, M., \& Fidge, C. (2011) Resource allocation and scheduling of multiple composite web services in cloud computing using cooperative coevolution genetic algorithm. In: Lu BL., Zhang L., Kwok J. (eds) Neural Information Processing. ICONIP 2011. Lecture Notes in Computer Science, vol. 7063. Springer, Berlin, Heidelberg.

Chen, X., Wang, H., Ma, Y., Zheng, X., \& Guo, L. (2020). Self-adaptive resource allocation for cloud-based software services based on iterative QoS prediction model. Future Generation Computer Systems, 105, 287-296.

Delamare, S., Fedak, G., Kondo, D., \& Lodygensky, O. (2012, June). SpeQuloS: a QoS service for BoT applications using best effort distributed computing infrastructures. In Proceedings of the 21st International Symposium on High-Performance Parallel and Distributed Computing (pp. 173-186). https://doi.org/10.1145/2287076.2287106.

Dewangan, B.K., Agarwal, A., \& Pasricha, A. (2016, October). Credential and security issues of cloud service models. In 2016 2nd International Conference on Next Generation Computing Technologies $(N G C T)$ (pp. 888-892). IEEE. Dehradun, India.

Dewangan, B.K., Agarwal, A., Venkatadri, M., \& Pasricha, A. (2018, December). Autonomic cloud resource management. In 2018 Fifth International Conference on Parallel, Distributed and Grid Computing $(P D G C)$ (Pp. 138-143). IEEE. Solan, Himachal Pradesh, India

Dewangan, B.K., Agarwal, A., Venkatadri, M., \& Pasricha, A. (2019). Self-characteristics-based EnergyEfficient Resource Scheduling for Cloud. Procedia Computer Science, 152, 204-211.

Kertesz, A., Kecskemeti, G., \& Brandic, I. (2011, February). Autonomic SLA-aware service virtualization for distributed systems. In 2011 19th International Euromicro Conference on Parallel, Distributed and Network-Based Processing (Pp. 503-510). IEEE. Ayia Napa, Cyprus. 
International Journal of Mathematical, Engineering and Management Sciences

Vol. 5, No. 4, 758-768, 2020

https://doi.org/10.33889/IJMEMS.2020.5.4.060

Levitin, G., Xing, L., \& Xiang, Y. (2020). Optimization of time constrained N-version programming service components with competing task execution and version corruption processes. Reliability Engineering \& System Safety, 193, 106666.

Mirjalili, S. (2015). The ant lion optimizer. Advances in Engineering Software, 83, 80-98.

Moschakis, I.A., \& Karatza, H.D. (2011, June). Performance and cost evaluation of gang scheduling in a cloud computing system with job migrations and starvation handling. In 2011 IEEE Symposium on Computers and Communications (ISCC) (Pp. 418-423). IEEE. Kerkyra, Greece.

Oprescu, A.M., \& Kielmann, T. (2010, November). Bag-of-tasks scheduling under budget constraints. In 2010 IEEE Second International Conference on Cloud Computing Technology and Science (Pp. 351359). IEEE. Indianapolis, IN, USA.

Reig, G., Alonso, J., \& Guitart, J. (2010, July). Prediction of job resource requirements for deadline schedulers to manage high-level SLAs on the cloud. In 2010 Ninth IEEE International Symposium on Network Computing and Applications (Pp. 162-167). IEEE. Cambridge, MA, USA.

Su, S., Li, J., Huang, Q., Huang, X., Shuang, K., \& Wang, J. (2013). Cost-efficient task scheduling for executing large programs in the cloud. Parallel Computing, 39(4-5), 177-188.

Sutar, S.G., Mali, P.J., \& More, A.Y. (2020). Resource utilization enhancement through live virtual machine migration in cloud using ant colony optimization algorithm. International Journal of Speech Technology, 23(1), 79-85.

Van Den Bossche, R., Vanmechelen, K., \& Broeckhove, J. (2010, July). Cost-optimal scheduling in hybrid iaas clouds for deadline constrained workloads. In 2010 IEEE 3rd International Conference on Cloud Computing (Pp. 228-235). IEEE. Miami, FL, USA.

Van Den Bossche, R., Vanmechelen, K., \& Broeckhove, J. (2013). Online cost-efficient scheduling of deadline-constrained workloads on hybrid clouds. Future Generation Computer Systems, 29(4), 973985 .

Xu, M., Cui, L., Wang, H., \& Bi, Y. (2009, August). A multiple QoS constrained scheduling strategy of multiple workflows for cloud computing. In 2009 IEEE International Symposium on Parallel and Distributed Processing with Applications (pp. 629-634). IEEE. Chengdu, China. 